\title{
The Heat Transfer Characteristics of Cooling Fan with Electromagnetic Driven Oscillating Foils
}

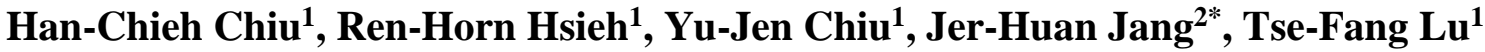 \\ ${ }^{1}$ Department of Mechanical Engineering, Taipei City University of Science and Technology, Pei-Tou, Taipei 112, Taiwan \\ hcchiu@tpcu.edu.tw; zhshieh@tpcu.edu.tw; yjchiu@tpcu.edu.tw \\ ${ }^{2}$ Department of Mechanical Engineering/Battery Research Center of Green Energy, Ming Chi University of Technology, \\ Taishan, New Taipei City 243, Taiwan \\ jhjang@mail.mcut.edu.tw
}

\section{Extended Abstract}

Electronic devices require effective and available cooling systems. Various designs of cooling systems have been proposed. For instance, high heat flux devices use phase change liquid cooling systems. However, most electronic devices use forced convection electric fan cooling because of cost issues. Recently, some researchers [1-4] have investigated piezoelectric fans for cooling systems with small size and low power consumption. Chiu et al. [5] studied a bio-mimetic oscillating foil driven by a grooved cam for the cooling of heat sinks. In this paper, a novel design of cooling fan with electromagnetic driven oscillating foil has been introduced and the characteristics of the cooling fan are under investigation.

In this study, the heat transfer from a hot surface with forced convective heat transfer using cooling fan with electromagnetic driven oscillating foil is discussed. A solenoid was excited with alternating current to actuate the magnet mounting on the rectangular PET foil. The rotational axis of the foil was placed perpendicular to the hot surface of the heat source. Air flow was induced by the foil motion and resulted in forced convective heat transfer on the heated surface. The surface temperature on the center of the heated surface (junction temperature) was measured for various current frequencies and foil locations. The effective thermal resistance was estimated through the difference between the junction temperature and the ambient temperature divided by the heat flux input.

In the experiment, the parameters include foil location, oscillating frequency, and foil length. The characteristics of the convective heat transfer depend on the oscillating frequency as well as the length of the foil. The frequencies for the study were set from $30 \mathrm{~Hz}$ to $110 \mathrm{~Hz}$ with increments of $20 \mathrm{~Hz}$. The applied current was set at $0.4 \mathrm{~A}$. The foil location, which was the distance between the foil tip and the center of the heated surface, ranged from 0 to $20 \mathrm{~mm}$. The results showed that the lowest thermal resistance was approximately $13.5^{\circ} \mathrm{C} / \mathrm{W}$ at a frequency of $50 \mathrm{~Hz}$. The oscillating amplitude decreased with the increase in oscillating frequency. Also, the cooling performance depended on the frequency as well as the flapping amplitude. It also found that higher oscillating frequencies than $50 \mathrm{~Hz}$ gave rise to higher thermal resistance. This was probably due to the smaller amplitude. In addition, the cooling performance at the resonant frequency was found to be significantly better than other frequencies.

\section{References}

[1] S. F. Liu, R. T. Huang, W. J. Sheu, and C. C. Wang, "Heat transfer by a piezoelectric fan on a flat surface subject to the influence of horizontal/vertical arrangement," Int. J. Heat Mass Trans., vol. 52, pp. 2565-2570, 2009.

[2] H. K. Ma, H. C. Su, C. L. Liu, and W. H. Ho, "Investigation of a piezoelectric fan embedded in a heat sink," Int. Comm. Heat Mass Trans., vol. 39, pp. 603-609, 2012.

[3] H. K. Ma, L. K. Tan, Y. T. Li, and C. L. Liu, "Optimum thermal resistance of the multiple piezoelectric-magnetic fan system,” Int. Comm. Heat Mass Trans., vol. 55, pp. 77-83, 2014.

[4] C. N. Lin and J. S. Leu, "Analysis of heat transfer and flow characteristics for the arrangement of piezoelectric fan on heated cylinder," Heat Transfer Eng., vol. 36, pp. 1525-1539, 2015.

[5] H. C. Chiu, R. H. Hsieh, Y. J. Chiu, J. H. Jang, and W. C. Lin, "Experimental study on the heat transfer of heat sink with bio-mimetic oscillating foil," Int. Comm. Heat Mass Trans., vol. 68, pp. 130-136, 2015. 